\title{
Two cobalt metal-organic frameworks constructed from mixed ligands and their magnetic properties
}

\author{
XIAO-HUAN QIN*, JING HUA and HUA-HONG ZOU*(D \\ State Key Laboratory for Chemistry and Molecular Engineering of Medicinal Resources, School of Chemistry \\ and Pharmacy, Guangxi Normal University, Guilin 541004, People's Republic of China \\ E-mail: gxnuchem@foxmail.com; 2667993910@qq.com
}

MS received 7 January 2019; revised 27 March 2019; accepted 23 April 2019; published online 21 June 2019

\begin{abstract}
The reaction of the polycarboxylic acid ligand tri(2-carboxyethyl)-isocyanurate $\left(\mathrm{H}_{3} \mathrm{~L}_{1}\right)$, polyazo bridged ligand $1 \mathrm{H}$-3-(3-pyridyl)-5-(4-pyridyl)-1,2,4-triazole $\left(\mathrm{L}_{2}\right), \mathrm{CoCl}_{2} \cdot 6 \mathrm{H}_{2} \mathrm{O}$, and different organic solvents lead to the formation of two novel coordination complexes: $\left[\mathrm{Co}_{3}\left(\mathrm{~L}_{1}\right)_{2}\left(\mathrm{~L}_{2}\right)_{2}\left(\mathrm{H}_{2} \mathrm{O}\right)_{4}\right]_{\mathrm{n}} \quad(\mathbf{1})$, $\left[\mathrm{Co}_{3}\left(\mathrm{~L}_{1}\right)_{2}\left(\mathrm{~L}_{2}\right)_{2}\left(\mathrm{H}_{2} \mathrm{O}\right)_{2}\right]_{\mathrm{n}} \cdot 2 \mathrm{nH}_{2} \mathrm{O}(\mathbf{2})$. Two complexes were prepared under solvothermal conditions and characterized by the single-crystal X-ray diffraction, thermal analysis, element analysis, X-ray powder diffraction, and IR spectra. Single-crystal X-ray analysis reveals that complex $\mathbf{1}$ features a three-dimensional framework and $\mathbf{2}$ is a two-dimensional layer structure. Variable-temperature magnetic susceptibility measurements show that both complexes display anti-ferromagnetic interactions between adjacent $\mathrm{Co}(\mathrm{II})$ centers.
\end{abstract}

Keywords. Co-ligands; solvothermal methods; crystal structures; magnetic properties.

\section{Introduction}

Co-based metal-organic frameworks (MOFs) have attracted much attention because of their fascinating architectures, special chemical and physical properties, and potential applications in many fields, such as catalysis, adsorption and separation of gas, magnetic, luminescence and so on. ${ }^{1-6}$ The judicious choice of the organic ligands are very important for the design and synthesis of MOFs. ${ }^{7-11}$ Polycarboxylic acids and polyazo bridged ligands have been extensively employed for constructing MOFs in previous studies. ${ }^{14,15}$ Of course, there are many factors that determine the final structures, such as the coordination geometry of metal ions, coordination mode of ligands, the ratio of metal-ligands, temperature, solvents, time of reaction and so on. ${ }^{12-15}$

Tri(2-carboxyethyl)-isocyanurate $\left(\mathrm{H}_{3} \mathrm{~L}_{1}\right)$, as flexible polycarboxylic acid ligand possesses many interesting characteristics: three highly flexible $-\mathrm{CH}_{2} \mathrm{CH}_{2} \mathrm{COOH}$ arms, three secondary functional groups $(-\mathrm{C}=\mathrm{O})$, various coordination modes, similarly aromatic and so

\footnotetext{
*For correspondence
}

on. The flexible tripodal multidentate carboxylate ligands, tri(2-carboxyethyl)-isocyanurate $\left(\mathrm{H}_{3} \mathrm{~L}_{1}\right)$ was chosen based on the following consideration: (1) the multidentate $\mathrm{H}_{3} \mathrm{~L}_{1}$ ligand with highly flexible arms has conformational flexibility and can adopt different coordination modes and diverse configurations via oxygen donors; (2) the flexible $\mathrm{H}_{3} \mathrm{~L}_{1}$ ligand has unique features and may facilitate the formation of novel unusual network topologies; (3) the flexible $\mathrm{H}_{3} \mathrm{~L}_{1}$ ligand is likely to coordinate with $3 \mathrm{~d}$ and $4 \mathrm{f}$ metal ions to assemble multinuclear or high-dimensional interpenetrating frameworks; ${ }^{16-20}$ (4) the flexible $\mathrm{H}_{3} \mathrm{~L}_{1}$ ligand has been relatively well-known and its metal-organic frameworks has shown interesting coordination architectures. ${ }^{10}$ The self-assembly between metal ions and flexible ligands has also proven to be the most effective synthetic design to achieve metal-organic frameworks. ${ }^{12}$

For novel structures and excellent properties, we introduce the secondary ligand, $1 H$-3-(3-pyridyl)-5-(4pyridyl)-1,2,4-triazole $\left(\mathrm{L}_{2}\right)$ as an assistant ligand, which has a bent backbone: the angle subtended at the center of the five-membered heterocyclic spacer and two pyridyl $\mathrm{N}$-donors is $143^{\circ}$, is a typical flexible linker. ${ }^{21-23}$ Herein, 

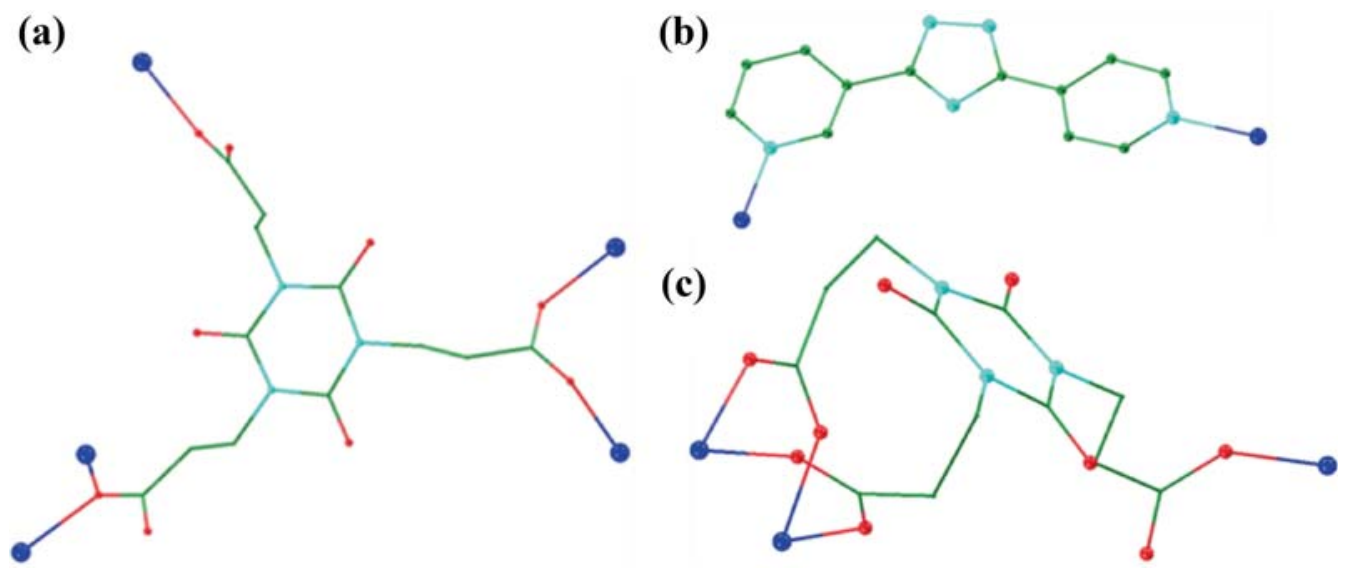

Scheme 1. The coordination modes of $\mathrm{L}_{1}$ (a for compound $\mathbf{1}, \mathrm{c}$ for compound 2 ) and $\mathrm{L}_{2}$ (b).

we designed and efficiently synthesized two new trinuclear $\mathrm{Co}(\mathrm{II})$-based MOFs: $\left[\mathrm{Co}_{3}\left(\mathrm{~L}_{1}\right)_{2}\left(\mathrm{~L}_{2}\right)_{2}\left(\mathrm{H}_{2} \mathrm{O}\right)_{4}\right]_{\mathrm{n}}(\mathbf{1})$ and $\left[\mathrm{Co}_{3}\left(\mathrm{~L}_{1}\right)_{2}\left(\mathrm{~L}_{2}\right)_{2}\left(\mathrm{H}_{2} \mathrm{O}\right)_{2}\right]_{\mathrm{n}} \cdot 2 \mathrm{nH}_{2} \mathrm{O}(2)$, and analysed their crystal structures. The coordination modes of $\mathrm{L}_{1}$ in two compounds are different (Scheme 1). The magnetic properties also have been discussed.

\section{Experimental}

\subsection{Materials and physical measurements}

The ligand $1 H$-3-(3-pyridyl)-5-(4-pyridyl)-1,2,4-triazole $\left(\mathrm{L}_{2}\right)$ was performed as previously reported, ${ }^{24}$ all other chemicals were commercially available and used as received without any further purification. IR spectra were recorded in the range of $4000-400 \mathrm{~cm}^{-1}$ on Perkin-Elmer Spectrum One FT/IR spectrometer using a $\mathrm{KBr}$ pellet. Elemental analysis for C, H, N was carried out with a model 2400II, Perkin-Elmer elemental analyzer. Detailed $a c$ and $d c$ magnetic data were collected on a Quantum Design MPMS SQUID-XL-5 magnetometer using the crushed single-crystal sample. Diamagnetic corrections were made with Pascal's constants. ${ }^{25}$

\subsection{Synthesis of $\left[\mathrm{Co}_{3}\left(\mathrm{~L}_{1}\right)_{2}\left(\mathrm{~L}_{2}\right)_{2}\left(\mathrm{H}_{2} \mathrm{O}\right)_{4}\right]_{n}$}

A mixture containing $\mathrm{CoCl}_{2} \cdot 6 \mathrm{H}_{2} \mathrm{O}(47.6 \mathrm{mg}, 0.2 \mathrm{mmol}), \mathrm{L}_{2}$ (46.4 mg, $0.2 \mathrm{mmol}$ ), $\mathrm{H}_{3} \mathrm{~L}_{1}(69 \mathrm{mg}, 0.2 \mathrm{mmol}), \mathrm{NaOH}(20 \mathrm{mg}$, $0.5 \mathrm{mmol})$, water $(10 \mathrm{~mL})$ were sealed in a Teflon-lined stainless steel vessel $(23 \mathrm{~mL})$, which was heated at $140^{\circ} \mathrm{C}$ for three days and then cooled to room temperature at a rate of $5^{\circ} \mathrm{Ch}^{-1}$. Prismatic pink crystals of $\mathbf{1}$ were obtained and picked out, washed with distilled water and dried in air. Yield: $55 \%$ (based on $\mathrm{CoCl}_{2} \cdot 6 \mathrm{H}_{2} \mathrm{O}$ ). Elemental analysis for $\mathrm{C}_{48} \mathrm{H}_{50} \mathrm{Co}_{3} \mathrm{~N}_{16} \mathrm{O}_{22}$ (\%), calc.: C, 41.78; H, 3.65; N, 16.24. Found: C, 41.59; H, 3.85; N, 16.17. IR (KBr, $\left.\mathrm{cm}^{-1}\right): 3555(\mathrm{w}), 3436(\mathrm{~m}), 2354$ (w), 1685 (s), 1570 (s), 1470 (s), 1392 (s), 1247 (m), 1147 (w), $986(w), 877(w), 756(w), 706(m), 518(\mathrm{~s}), 451(w)$.

\subsection{Synthesis of $\left[\mathrm{Co}_{3}\left(\mathrm{~L}_{1}\right)_{2}\left(\mathrm{~L}_{2}\right)_{2}\left(\mathrm{H}_{2} \mathrm{O}\right)_{2}\right]_{n} \cdot 2 n \mathrm{H}_{2} \mathrm{O}$}

A mixture containing $\mathrm{CoCl}_{2} \cdot 6 \mathrm{H}_{2} \mathrm{O}(47.6 \mathrm{mg}, 0.2 \mathrm{mmol})$, $\mathrm{L}_{2}$ (46.4 mg, $\left.0.2 \mathrm{mmol}\right), \mathrm{H}_{3} \mathrm{~L}_{1}$ (69 mg, $\left.0.2 \mathrm{mmol}\right), \mathrm{NaOH}$ (20 mg, $0.5 \mathrm{mmol})$, water $(10 \mathrm{~mL})$ and ethanol $(2 \mathrm{~mL})$ were sealed in a Teflon-lined stainless steel vessel $(23 \mathrm{~mL})$, which was heated at $140^{\circ} \mathrm{C}$ for three days and then cooled to room temperature at a rate of $5^{\circ} \mathrm{C} \mathrm{h}^{-1}$. Light blue block crystals of 2 were obtained and picked out, washed with distilled water and dried in air. Yield: $46 \%$ (based on $\mathrm{CoCl}_{2} \cdot 6 \mathrm{H}_{2} \mathrm{O}$ ). Elemental analysis for $\mathrm{C}_{48} \mathrm{H}_{48} \mathrm{Co}_{3} \mathrm{~N}_{16} \mathrm{O}_{22}$ (\%), calcd: $\mathrm{C}, 41.84 ; \mathrm{H}, 3.51$; $\mathrm{N}, 16.27 \%$. Found: C, 41.65; H, 3.82; N, 16.13. IR (KBr, $\mathrm{cm}^{-1}$ ): $3551(\mathrm{w}), 3429(\mathrm{~m}), 2353(\mathrm{w}), 1686(\mathrm{~s}), 1571(\mathrm{~s})$, $1468(\mathrm{~s}), 1389(\mathrm{~s}), 1245(\mathrm{~m}), 1143(\mathrm{w}), 988(\mathrm{w}), 876(\mathrm{w}), 757$ (w), $705(\mathrm{~m}), 519(\mathrm{~s}), 450(\mathrm{w})$.

\section{$2.4 \quad X$-ray crystallography}

All data were collected on a Bruker Smart Apex-II CCD diffractometer equipped with graphite-monochromated Mo $\mathrm{K} \alpha$ radiation $(\lambda=0.71073 \AA$ ) at $293(2) \mathrm{K}$. Raw frame data were integrated with the SAINT program. The structures were solved by direct methods using SHELXTL and refined by full-matrix least squares on $F^{2}$ using SHELXTL. ${ }^{26}$ An empirical absorption correction was applied with the program SADABS. All non-hydrogen atoms were refined anisotropically. Complete crystallographic data have been deposited at the Cambridge Crystallographic Data Centre and may be obtained free of charge via www.cznc.cam.ac.uk/data request/cif. The crystallographic details are provided in Table 1 , and the selected bond distances and angles are listed in Table S1 (Supplementary Information).

\section{Results and Discussion}

\subsection{Description of the crystal structures}

3.1a $\left[\mathrm{Co}_{3}\left(L_{1}\right)_{2}\left(\mathrm{~L}_{2}\right)_{2}\left(\mathrm{H}_{2} \mathrm{O}\right)_{4}\right]_{n}(\mathbf{1})$ : Single-crystal Xray structural analysis shows that complex $\mathbf{1}$ is a $3 \mathrm{D}$ 


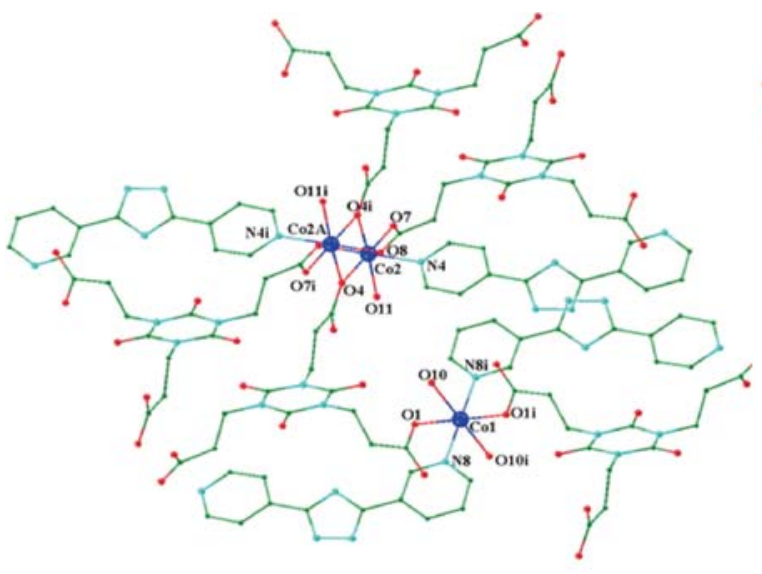

(a)

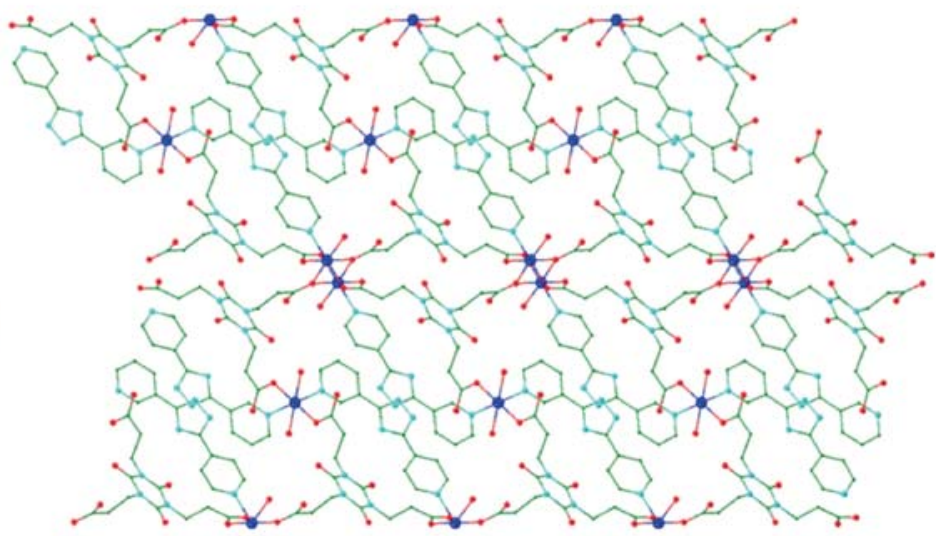

(b)

Figure 1. (a) The perspective view of the $\mathrm{Co}(\mathrm{II})$ coordination environments in the trimer of 1, C, green; O, red; N, light blue; Co, navy blue, all hydrogen atoms are omitted for clarity. (b) Partial perspective of the 2D crystal structure of $\mathbf{1}$ along the crystallographic a-axis direction.

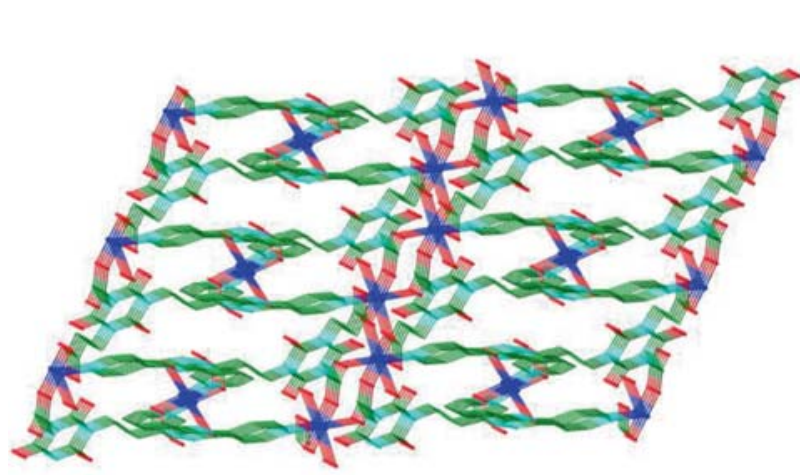

(a)

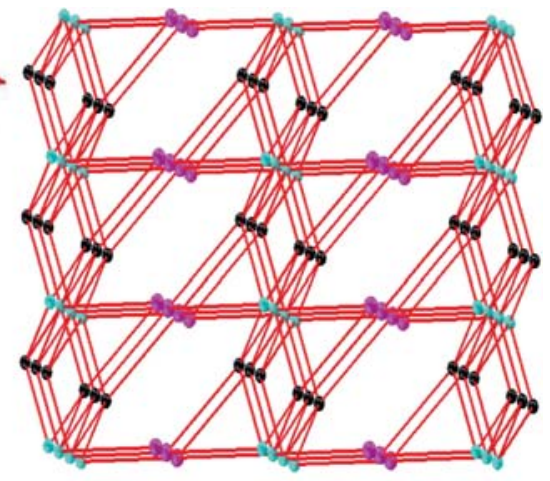

(b)

Figure 2. (a) Partial perspective of the 3D crystal structure in 1. (b) Partial perspective of topology structure in 1.

metal-organic framework. As illustrated in Figure 1a, the asymmetric unit contains one and a half cobalt ions, four coordinated water molecules, two bridged $\mathrm{L}_{2}$ ligands and two 3 -connected $\mathrm{L}_{1}$ ligands. The Co1 is coordinated by two oxygen atoms from two different carboxylates of $\mathrm{L}_{1}$ ligands $(\mathrm{O} 1$ and $\mathrm{O} 1 \mathrm{i}$, symmetry codes: i: 1-x, 1-y, 1-z) and two water molecules (O10 and $\mathrm{O} 10 \mathrm{i}$ ), and two nitrogen atoms from two $\mathrm{L}_{2}$ ligands (N8 and N8i). The Co2 is bound to four carboxylate oxygen atoms $(\mathrm{O} 4, \mathrm{O} 4 \mathrm{i}, \mathrm{O} 7, \mathrm{O} 8 \mathrm{i}$, the symmetry codes: $\mathrm{i}:-1+\mathrm{x},-1+\mathrm{y}, \mathrm{z})$, one pyridyl nitrogen atom $(\mathrm{N} 4)$ and one oxygen atom $(\mathrm{O} 11)$ from the coordinated water. The central Co2A lies on a crystallographic inversion center and is linked by two carboxylate oxygen atoms to the $\mathrm{Co} 2$ atoms. The trimer is further inter-linked by the coordinated $\mathrm{L}_{1}$ and $\mathrm{L}_{2}$ ligands resulting in a 2D layer structure (Figure $1 b$ ), the $\mathrm{L}_{1}$ ligand has a -3 charge with three carboxylate groups deprotonated in 1. And when $\mathrm{L}_{1}$ adopts $\mu_{5}-\kappa^{5} \mathrm{O}^{1}: \mathrm{O}^{2}: \mathrm{O}^{3}: \mathrm{O}^{4}, \mathrm{O}^{5}$ coor- dination fashion, adjacent trimer units get connected by $\mathrm{L}_{1}$ ligands into $3 \mathrm{D}$ MOF (Figure 2). Considering each tri-nuclear unit, $\mathrm{L}_{1}$, and $\mathrm{L}_{2}$ ligand as six-, three-, two-connecting nodes, the 3D structure of $\mathbf{1}$ topologically possesses a 6-connected uninodal primitive cubic with the point (Schläfli) symbol $3^{2} \cdot 5 \cdot 6^{3}$ calculated using TOPOS software (Figure 2). ${ }^{27}$ Many other compounds containing similar ligands have been reported, such as $\left\{\left[\mathrm{Zn}_{2}(\mathrm{~m}-\mathrm{BDC})_{2}\left(4,4^{\prime}-\mathrm{bpt}\right)\left(\mathrm{H}_{2} \mathrm{O}\right)_{3}\right]\right.$. $\left.\mathrm{H}_{2} \mathrm{O}\right\}_{n}$, which is obtained under similar hydrothermal conditions and shows bilayer motif with $8^{2} \cdot 10$ topology. ${ }^{27}$ Four Cd-based metal-organic frameworks are reported using different alkaline reagents and exhibiting different topology by $\mathrm{Bai}$ and $\mathrm{Zhu},{ }^{10}$ the results indicate that the alkaline reagents play a crucial role in the diversity of the structures and coordination modes of the $\mathrm{L}_{1}$ ligand. Han group reported two novel coordination polymers, $\left[\mathrm{Zn}_{3}\left(\mathrm{~L}_{1}\right)_{2}(\mathrm{bpy})_{1.5}\left(\mathrm{H}_{2} \mathrm{O}\right)_{2}\right] \cdot 6 \mathrm{H}_{2} \mathrm{O}$ and $\left[\mathrm{ZnNa}\left(\mathrm{L}_{1}\right)(\text { bpy })_{0.5}\left(\mathrm{H}_{2} \mathrm{O}\right)\right] \cdot 2 \mathrm{H}_{2} \mathrm{O}$, which feature 

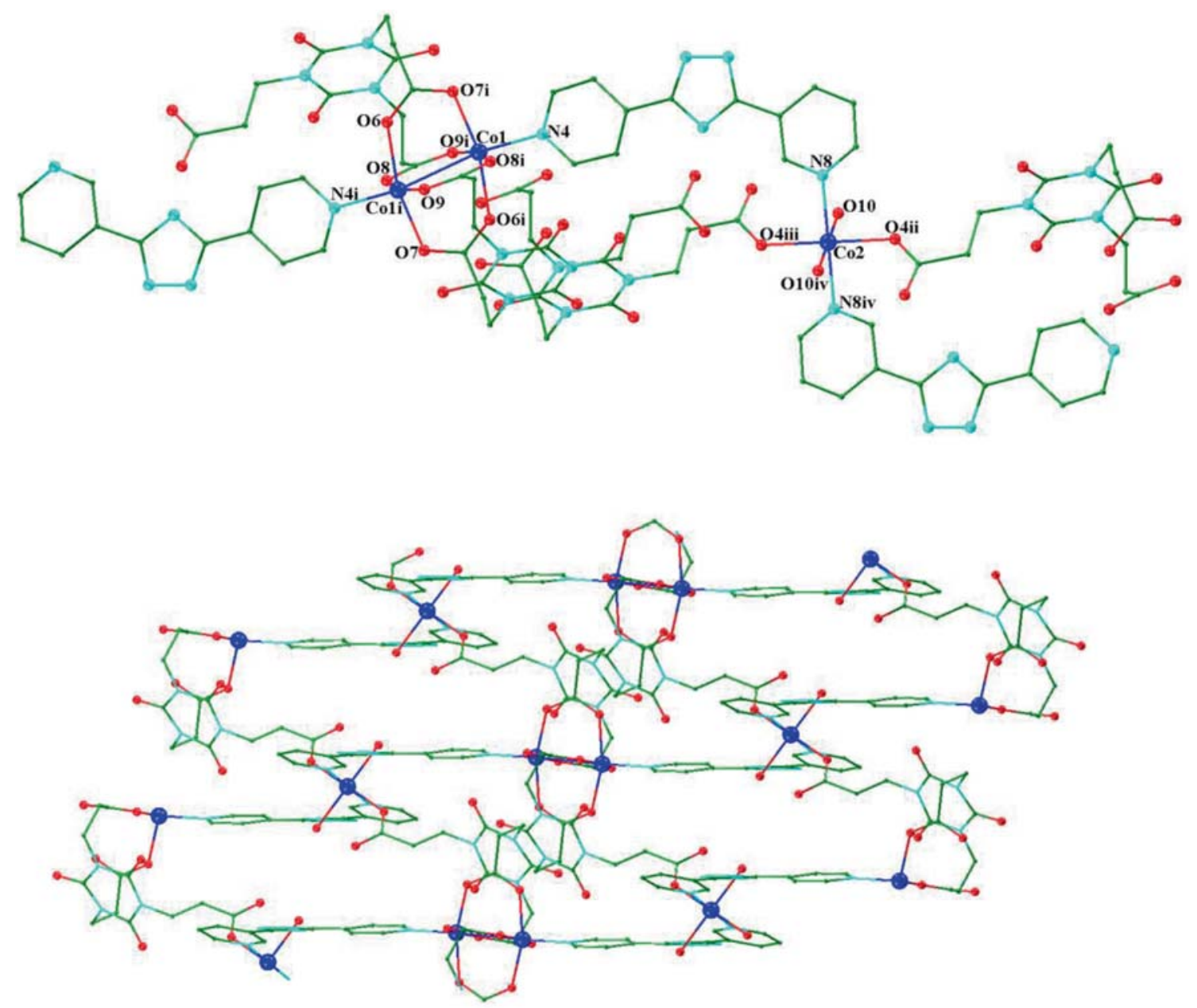

Figure 3. The perspective view of the $\mathrm{Co}(\mathrm{II})$ coordination environments in the trimer of $\mathbf{2}, \mathrm{C}$, green; $\mathrm{O}$, red; N, light blue; Co, navy blue, all hydrogen atoms are omitted for clarity (up); Partial perspective of the 2D crystal structure of $\mathbf{2}$ along the crystallographic c-axis direction (down).

a three-fold interpenetrating $(3,4,5)$-connected $3 \mathrm{D}$ network and a two-fold interpenetrating $(4,5)$-connected $\left(4^{3} .6^{3}\right)\left(4^{3} .6^{6} .8\right)$-fsx network, respectively. ${ }^{10}$

$3.1 \mathrm{~b} \quad\left[\mathrm{Co}_{3}\left(\mathrm{~L}_{1}\right)_{2}\left(\mathrm{~L}_{2}\right)_{2}\left(\mathrm{H}_{2} \mathrm{O}\right)_{2}\right]_{n} \cdot 2 n \mathrm{H}_{2} \mathrm{O}$ (2): Complex 2 is a $2 \mathrm{D}$ layer structure, the asymmetric unit also contains one and a half cobalt ions, two coordinated water molecules, two $\mathrm{L}_{1}$ ligands, two $\mathrm{L}_{2}$ ligands, and two solvent water molecules (Figure 3). The Co1 atoms are coordinated by four carboxylate oxygen atoms (O6i, O7i, O8i, O9i; symmetry codes: i: 2-x, 1-y, -z), one pyridyl nitrogen atoms (N4), and Co1A atom is in a symmetrical position. The $\mathrm{Co} 2$ atoms are bonded to two carboxylate oxygen atoms (O4ii and O4iii; the symmetry codes: ii: $1+x, y, z$; iii: $2-x .2-y, 1-z)$, two pyridyl nitrogen atoms (N8 and N8iv, the symmetry code: iv: 3-x, 2-y, 1-z), and two coordinated water (O10 and O10iv). Each $\mathrm{L}_{1}$ and $\mathrm{L}_{2}$ ligands adopt $\mu_{3}-\kappa^{5} \mathrm{O}^{1}, \mathrm{O}^{2}: \mathrm{O}^{3}, \mathrm{O}^{4}, \mathrm{O}^{5}$ and $\mu_{2}-\kappa^{2} \mathrm{~N}^{1}: \mathrm{N}^{2}$ coordination

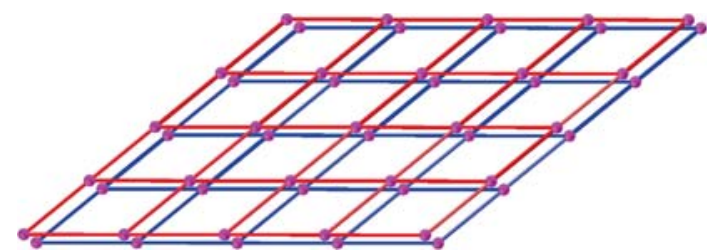

Figure 4. Partial perspective of the topology structure of complex 2.

fashion, respectively. Each trimer is inter-linked by the coordinated $\mathrm{L}_{1}$ and $\mathrm{L}_{2}$ ligands resulting in a $2 \mathrm{D}$ layer structure. Considering each tri-nuclear unit, $\mathrm{L}_{1}$, and $\mathrm{L}_{2}$ ligand as four-, two-, two-connecting nodes, the 2D structure of $\mathbf{2}$ topologically possesses a 4-connected network with the point (Schläfli) symbol $4^{4}$ calculated using TOPOS software (Figure 4). ${ }^{30}$

Although the formulas of these two compounds are similar, the coordination modes of the $\mathrm{L}_{1}$ ligand in $\mathbf{1}$ and 

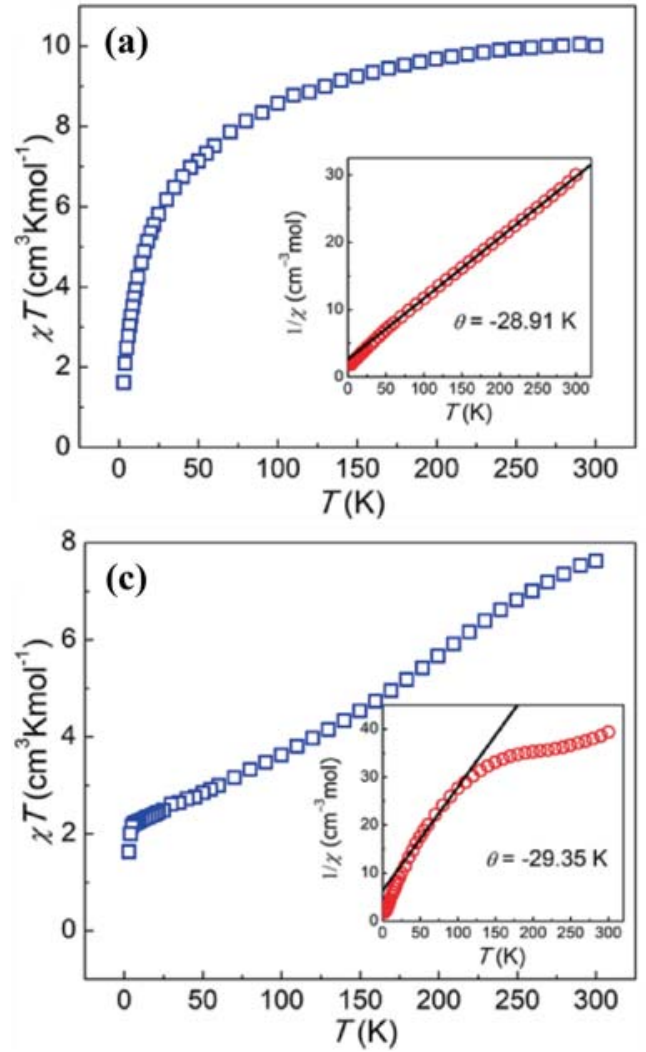

Figure 5. Temperature dependence of $\chi_{m} T$ magnetization (b) for $\mathbf{1}$, (d) for $\mathbf{2}$.

$\mathbf{2}$ are very different. For complex $\mathbf{1}$, one $\mathrm{L}_{1}$ ligand coordinated to five different $\mathrm{Co}^{2+}$ ions, and there are three $\mu_{1}-\mathrm{O}$ atoms and one $\mu_{2}-\mathrm{O}$ atom (Figure $1 \mathrm{c}$ ). But there are five $\mu_{1}-\mathrm{O}$ atoms coordinated three $\mathrm{Co}^{2+}$ ions in 2 (Figure 3b). On the other hand, there are four coordinated water molecules in $\mathbf{1}$, but two coordinated water molecules and two solvent water molecules in $\mathbf{2}$. These factors lead to different crystal structures.

\subsection{X-ray powder diffraction results}

To confirm whether the crystal structures are truly representative of the bulk materials, X-ray powder diffraction (XRPD) experiments have also been carried out for two complexes (Figure S1, Supplementary Information). The XRPD experimental and computer-simulated patterns of the corresponding complexes are shown in Figure 5. Although the experimental patterns have a few unindexed diffraction lines and some are slightly broadened in comparison with those simulated from the single crystal models, it can still be considered favourably that the bulk synthesized materials and the as-grown crystals are homogeneous for two complexes.
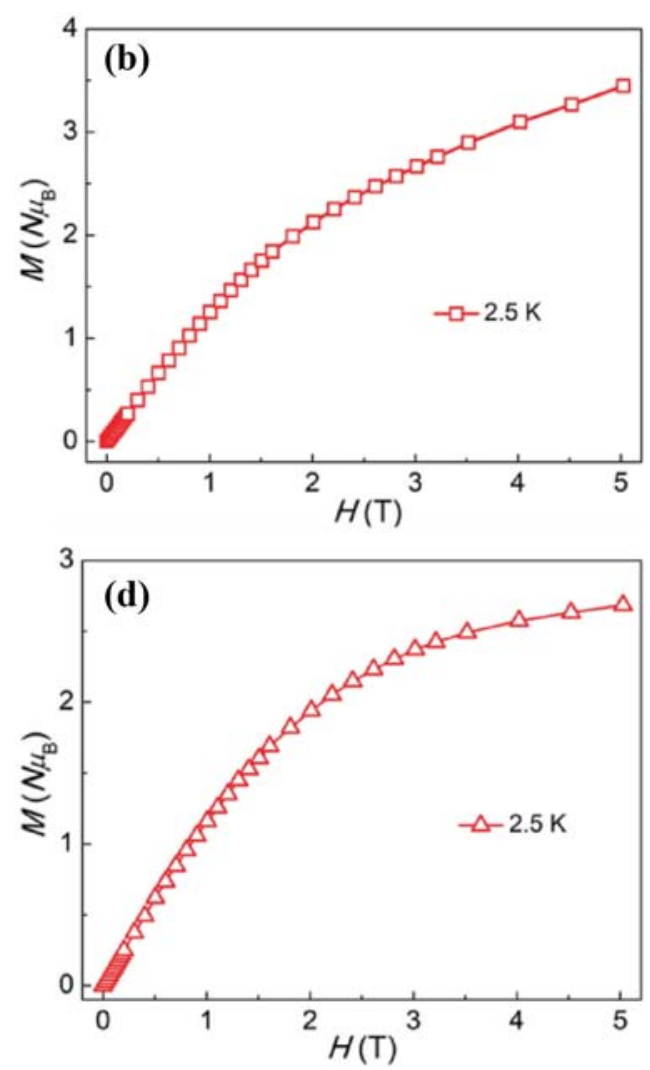

(a) for $\mathbf{1}$, (c) for $\mathbf{2}$, and field dependence of the

\subsection{Raman spectra}

Raman spectrums of complexes $\mathbf{1}$ and $\mathbf{2}$ in the lowfrequency region $\left(200-2000 \mathrm{~cm}^{-1}\right)$ are shown in Figure S2 (Supplementary Information). Their relative strong bands in $\mathbf{1}$ and $\mathbf{2}$ are observed when excited with the line at $514 \mathrm{~nm}$. The bands at 531 and $535 \mathrm{~cm}^{-1}$ in $\mathbf{1}$ and $\mathbf{2}$, are assigned to the bending and symmetric stretching vibrations of the framework Co-O-Co species, respectively. The peaks at 571,624 and $743 \mathrm{~cm}^{-1}$ for $\mathbf{1}$, and 570,657 and $741 \mathrm{~cm}^{-1}$ for 2 may be the wagging, twisting, and rocking modes of the coordinate water molecules, respectively. The COO symmetric (1413 and $1422 \mathrm{~cm}^{-1}$ ) and asymmetric stretching (1512 and $1507 \mathrm{~cm}^{-1}$ ) mode are for complexes $\mathbf{1}$ and $\mathbf{2}$, respectively. The bands 1622 and $1618 \mathrm{~cm}^{-1}$ in $\mathbf{1}$ and $\mathbf{2}$ are assigned to stretching vibrations of the carbonyl group, respectively. The rest of bands in $\mathbf{1}$ and $\mathbf{2}$ are assigned to vibration and breathing of ring. In the IR spectrum of 1 and 2, characteristic bands of carboxylic groups occur at 1570 and $1571 \mathrm{~cm}^{-1}$ for asymmetric stretching and at 1392 and $1389 \mathrm{~cm}^{-1}$ for the symmetric stretching, respectively. The comparatively low $\Delta$ value (178 and $199 \mathrm{~cm}^{-1}, \Delta=v_{\text {as }} \mathrm{COO}^{-}-v_{\mathrm{s}} \mathrm{COO}^{-}$) possibly indicates that $\mathrm{L}_{1}$ is bidentate and complete deprotonation of the 

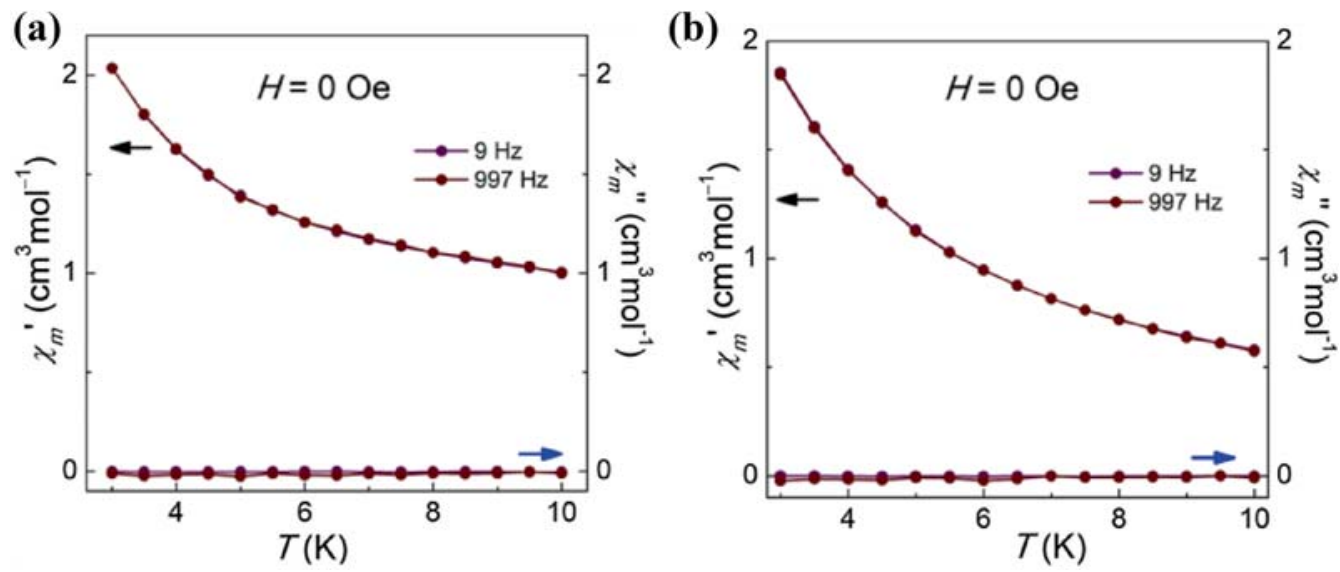

Figure 6. Temperature dependence of the $\chi^{\prime}$ and $\chi^{\prime \prime}$ ac-susceptibilities of $\mathbf{1}$ (a) and $\mathbf{2}$ under 0 Oe dc-field (b).

$\mathrm{H}_{3} \mathrm{~L}_{1}$ ligand upon reaction with metal ions in $\mathbf{1}$ and $\mathbf{2}$. The Raman and IR spectrum complement each other.

\subsection{Thermal analysis}

The thermal stability of these new crystalline materials has been investigated by thermogravimetric analysis experiments of mass loss (Figure S3, Supplementary Information). The TGA curve of $\mathbf{1}$ suggests that the first weight loss of $3.74 \%$ in the region of $30-270{ }^{\circ} \mathrm{C}$ (peak at $58^{\circ} \mathrm{C}$ ) corresponds to the release of the three coordinated water (calculated $3.92 \%$ ). The residual framework starts to decompose from $271^{\circ} \mathrm{C}$ (peaks at $281^{\circ} \mathrm{C}$, $427^{\circ} \mathrm{C}$ ) with a series of complicated weight losses and does not stop until heating ends at $1000{ }^{\circ} \mathrm{C}$. For the TGA curve of $\mathbf{2}$, there are several mass loss steps, the first weight loss of $6.26 \%$ in the region of $30-280^{\circ} \mathrm{C}$ (peak at $48^{\circ} \mathrm{C}$ ) corresponds to the loss of two coordinated water and two free water (calculated 5.22\%). The residual framework starts to decompose (peaks at 310 , $456,504,630$, and $674^{\circ} \mathrm{C}$ ) from $281^{\circ} \mathrm{C}$ and ended at $1200^{\circ} \mathrm{C}$.

\subsection{Magnetic properties}

The direct current magnetic susceptibilities of $\mathbf{1}$ and 2 were measured at the temperature range 300-2 K under 1000 Oe field (Figure 5). The $\chi_{m} T$ values at room temperature were $10.00 \mathrm{~cm}^{3} \cdot \mathrm{K} \cdot \mathrm{mol}^{-1}$ for $\mathbf{1}$ and $7.63 \mathrm{~cm}^{3} \cdot \mathrm{K} \cdot \mathrm{mol}^{-1}$ for 2 , which were larger than the expected value of $5.625 \mathrm{~cm}^{3} \cdot \mathrm{K} \cdot \mathrm{mol}^{-1}$ for three uncorrelated $\mathrm{Co}^{2+}$ ions $(S=3 / 2)$ and is indicative of the orbital contribution of octahedral $\mathrm{Co}$ (II). On lowering the temperature, $\chi_{m} T$ decreases marginally up to $c a$. $100 \mathrm{~K}(\mathbf{1})$ and $150 \mathrm{~K}(\mathbf{2})$ before undergoing a shallow minima at approximately $48 \mathrm{~K}$ (1) and $33 \mathrm{~K}(\mathbf{2})$, and then quickly decreases to approximately 1.61 (1) and $1.63(2) \mathrm{cm}^{3} \cdot \mathrm{K} \cdot \mathrm{mol}^{-1}$ at $3 \mathrm{~K}$, respectively. The analysis of $\chi_{m}^{-1}$ versus $T$ at high temperature based on the Curie-Weiss law yielded estimated Curie constants $(C)$ of 11.05 and $4.63 \mathrm{~cm}^{3} \cdot \mathrm{K} \cdot \mathrm{mol}^{-1}$ for $\mathbf{1}$ and 2, respectively. ${ }^{27}$ The Weiss constants $(\theta)$ of $\mathbf{1}$ and $\mathbf{2}$ are $-28.91 K$ and $-29.35 K$ (Figure $5 \mathrm{a}$ and $5 \mathrm{c}$ ). The $C$ values are typical for octahedral $\mathrm{Co}(\mathrm{II})$, and negative $\theta$ value indicates intracluster antiferromagnetic coupling. ${ }^{31}$ Obviously, the configurations of $\mathrm{Co}$ (II) tune the magnetic anisotropy by spin-orbit coupling to contribute to the overall magnetic behavior. Similar situations have been observed in other examples. ${ }^{27-29}$ The $M-H$ for each complex was measured under the maximum magnetic field at $5 \mathrm{~T}$ (Figure $5 \mathrm{~b}$ and $5 \mathrm{~d}$ ). The magnetization curves of $\mathbf{1}$ and $\mathbf{2}$ under low magnetic field flux intensities rapidly increase before slowly increasing to the values of 3.45 and $2.68 N \mu_{B}$ under $5 \mathrm{~T}$ at $2 \mathrm{~K}$, indicates that $\mathbf{1}$ and $\mathbf{2}$ exhibits considerable magnetic anisotropy, which is comparable to the previously reported analogue $\mathrm{Co} 3$ systems with mixed ligands bridges. ${ }^{28-32}$

The dynamic magnetic properties of $\mathbf{1}$ and $\mathbf{2}$ were investigated by measurements of AC magnetic susceptibility. Complexes $\mathbf{1}$ and $\mathbf{2}$ do not display any $\chi^{\prime \prime}$ signal at zero static magnetic field over the frequency range of $\mathrm{Hz} 9$ and $997 \mathrm{~Hz}$ down to $2 \mathrm{~K}$. No imaginary component is observed (Figure 6).

\section{Conclusions}

Using the solvothermal method, two MOFs based on cobalt and mixed organic ligands have been synthesized. And single-crystal X-ray analysis suggests that the structure of $\mathbf{1}$ feature a $3 \mathrm{D}$ MOFs and the $\mathbf{2}$ is a 2D framework. AC susceptibility studies indicate that $\mathbf{1}$ and $\mathbf{2}$ display no out-of-phase component of 
the ac-susceptibilities, and no imaginary component is observed. This study provides an example of the synthesis of 3D MOFs by simply changing the reaction solvent to construct complexes with different structures.

\section{Supplementary Information (SI)}

Crystallographic data are available free of charge from The Director, CCDC, 12 Union Road, Cambridge, CB2 1EZ, UK (Fax: +44-1223-336033; Email: deposit@ccdc.cam.ac.uk or http://www.ccdc.cam.ac.uk) upon request, quoting deposition number CCDC 1883706 for $\mathbf{1}$, and 1883707 for $\mathbf{2}$. Supplementary Information is available at www.ias.ac.in/ chemsci.

\section{Acknowledgements}

This work was supported by the National Natural Science Foundation of China (No. 21601038), Guangxi Natural Science Foundation (No. 2016GXNSFAA380085), the Project of Improving the Basic Ability of Young and Middleaged Teachers in Guangxi Higher Education Institutions (2017KY0069).

\section{References}

1. Zhang X, Huang Y Y, Lin Q P, Zhang J Y and Yao G 2013 Using alkaline-earth metal ions to tune structural variations of 1,3,5-benzenetricarboxylate coordination polymers Dalton Trans. $\mathbf{4 2} 2294$

2. Li J R, Sculley J and Zhou H C 2012 Metal-organic frameworks for separations Chem. Rev. 112869

3. Zou H H, He Y P, Gui L C and Liang F P 2011 A new 8-connected porous coordination polymer: crystal structure and selective adsorption properties CrystEngComm 133325

4. Huang F P, Bian H D, Yu Q, Tian J L, Liang H, Yan S P, Liao D Z and Cheng P 2011 Coordination assemblies of $\mathrm{Co}^{\mathrm{II}} / \mathrm{Ni}^{\mathrm{II}} / \mathrm{Mn}^{\mathrm{II}} / \mathrm{Zn}^{\mathrm{II}}$ with $1,1^{\prime}$-biphenyl-2, 2' dicarboxylic acid and three positional isomeric ligands: structural diversity and properties CrystEngComm $\mathbf{1 3}$ 6538

5. Yang W T, Guo M, Yi F Y and Sun Z M 2012 Cryst. Growth Des. 125529

6. Chowdhury B, Bhowmik B, Sahu A, Joshi M, Paul S, Choudhury A R and Biswas B 2018 Phenoxazinone synthase and antimicrobial activity by a bis(1,3-diamino2-propanolate) cobalt(III) complex J. Chem. Sci. 130161

7. Lian X, Fang Y, Joseph E, Wang Q, Li J, Banerjee S, Lollar C, Wang X and Zhou H C 2017 Enzyme-MOF (metal-organic framework) composites Chem. Soc. Rev. 463386

8. Tan Y X, Zhang Y, He Y P and Zheng Y J 2014 Microporous metal-organic layer built from pentanuclear tetrahedral units: gas sorption and magnetism New J. Chem. 385272

9. Luo J, Hong M, Wang R, Cao R, Han L, Yuan D, Lin Z and Zhou Y 2003 A novel bilayer cobalt(II) organic framework with nanoscale channels accommodating large organic molecules Inorg. Chem. 424486

10. (a) Qin X H, Gui L C, Yao P F, Zou H H, Li H Y and Huang F P 2019 Synthesis, structures, and properties of a family of 3d-based MOFs constructed from mixed ligands Trans. Met. Chem. 44 31; (b) Bai Y L, Xu L Z, Bao X L, Hou C Y, Zhao Y M and Zhu S R 2016 Alkaline reagent-induced structural diversity of four metal-organic frameworks based on a flexible bicarboxylate ligand CrystEngComm 18 2347; (c) Han Z B and Zhang G X 2010 Solvothermal synthesis of two unique metal-organic frameworks: a 3-fold interpenetrating $(3,4,5)$-connected network and a 2-fold interpenetrating (4,5)-connected network CrystEng Comm 12348

11. He Y P, Tan Y X and Zhang J 2012 Deliberate design of a neutral heterometallic organic framework containing a record 25-fold interpenetrating diamondoid network CrystEngComm 146359

12. He Y, Li B, O'Keeffec M and Chen B 2014 Multifunctional metal-organic frameworks constructed from meta-benzenedicarboxylate units Chem. Soc. Rev. 43 5618

13. Qin L, Wang Z, Wang T, Zheng H and Chen J 2014 Coligand-directed synthesis of five $\mathrm{Co}(\mathrm{II}) / \mathrm{Ni}(\mathrm{II})$ coordination polymers with a neutral tetradentate ligand: syntheses, crystal structures, and properties Dalton Trans. 4312528

14. Lin R G, Lin R B and Chen B 2017 A microporous metal-organic framework for selective $\mathrm{C}_{2} \mathrm{H}_{2}$ and $\mathrm{CO}_{2}$ separation J. Solid State Chem. 252138

15. Chen G, He Y, Zhang S and Zhang J 2018 Tuning a layer to a three-dimensional cobalt-tris $\left(4^{\prime}\right.$ carboxybiphenyl)amine framework by introducing potassium ions Inorg. Chem. Commun. 9065

16. Song T Q, Dong J, Gao H L and Cui J Z 2017 Three coordination polymers based on $\mathrm{M}_{2}(\mathrm{M}=\mathrm{Co}, \mathrm{Ni}$ and $\mathrm{Zn})$ clusters: Structures, magnetic and fluorescent properties Inorg. Chim. Acta 466393

17. Huang F P, Yang Z M, Yao P F, Yu Q, Tian J L, Bian H D, Yan S P, Liao D Z and Cheng P 2013 Coordination assemblies of the $\mathrm{Cd}^{\mathrm{II}}-\mathrm{BDC} / \mathrm{bpt}$ mixed-ligand system: positional isomeric effect, structural diversification and luminescent properties CrystEngComm 152657

18. Ma L, Abney C and Lin W 2009 Enantioselective catalysis with homochiral metal-organic frameworks Chem. Soc. Rev. 381248

19. Yao C, Wang W, Zhang S R, Li H Y, Xu Y H, Su Z $\mathrm{M}$ and Che G B 2018 A multifunctional microporous metal-organic framework: efficient adsorption of iodine and column-chromatographic dye separation RSC Adv. 836400

20. Amayuelas E, Fidalgo-Marijuán A, Bazán B, Urtiaga M K, Barandika G and Arriortua M I 2017 Highly thermally stable heterogeneous catalysts: study of OD and 3D porphyrinic MOFs CrystEngComm 19 7244

21. Tan H, Chen Q, Sheng Y, Li X and Liu H 2018 Guest-induced reversible crystal-to-amorphous-tocrystal transformation in a Co(II)-based metal-organic framework CrystEngComm $\mathbf{2 0} 6828$

22. Du M, Jiang X J and Zhao X J 2006 Controllable assembly of metal-directed coordination polymers under 
diverse conditions: A case study of the $\mathrm{M}^{\mathrm{II}}-\mathrm{H}_{3} \mathrm{tma} / \mathrm{Bpt}$ mixed-ligand system Inorg. Chem. 453998

23. Zhang C, Xiao Y, Qin Y, Sun Q and Zhang S 2018 A novel highly efficient adsorbent $\left\{\left[\mathrm{Co}_{4}(\mathrm{~L})_{2}\left(\mu_{3}-\mathrm{OH}\right)_{2}\left(\mathrm{H}_{2} \mathrm{O}\right)_{3}(4,4 \text { '-bipy })_{2}\right] \cdot\left(\mathrm{H}_{2} \mathrm{O}\right)_{2}\right\}_{n}$ : synthesis, crystal structure, magnetic and arsenic (V) absorption capacity J. Solid State Chem. 26122

24. Liu H, Gao J, Maynard L, Saito Y D and Kool E T 2004 Toward a new genetic system with expanded dimensions: size-expanded analogues of deoxyadenosine and thymidine J. Am. Chem. Soc. 1261102

25. Boudreaux E A and Mulay J N 1976 Theory and Application of Molecular Diamagnetism (New York: Wiley)

26. Sheldrick G M 2008 A short history of SHELX Acta Cryst. A64 112

27. Huang F P, Li H Y, Yu Q, Bian H D, Tian J L, Yan S P, Liao D Z and Cheng P 2012 Co(II)/Ni(II) coordination polymers incorporated with a bent connector: crystal structures and magnetic properties CrystEngComm 14 4756

28. (a) Mahata P, Smart D and Natarajan S 2010 Magnetic behaviour in metal-organic frameworks-Some recent examples J. Chem. Sci. 122 19; (b) Zhang Y J, Yang J
H, Wang J J and Chen J 2016 Synthesis, crystal structure and magnetic properties of a Cobalt(II) complex with quinoxaline-substituted nitronyl nitroxide radicals Chin. J. Struct. Chem. 35959

29. (a) Natarajan S, Mahata P and Smart D 2012 The relevance of metal organic frameworks (MOFs) in inorganic materials chemistry J. Chem. Sci. 124 339; (b) Xiong L. N, Liu Q Y and Wang Y L 2016 Crystal structure and magnetic properties of a two-dimensional compound. $\left.\left.\left\{\left(\mathrm{Me}_{2} \mathrm{NH}_{2}\right)_{4}\right] \mathrm{Co}(\mathrm{SIP})_{2}\right] \cdot 2(\mathrm{DMF}) \cdot 3\left(\mathrm{H}_{2} \mathrm{O}\right)\right\}_{\mathrm{n}}$ Chin. J. Struct. Chem. 351231

30. Ouellette W, Prosvirin A V, Whitenack K, Dunbar K R and Zubieta J 2009 A thermally and hydrolytically stable microporous framework exhibiting single-chain magnetism: structure and properties of $\left[\mathrm{Co}_{2}\left(\mathrm{H}_{0.67} \mathrm{bdt}\right)_{3}\right]$. $20 \mathrm{H}_{2} \mathrm{O}$ Angew. Chem. Int. Ed. 482140

31. Chen $\mathrm{G} \mathrm{H}$, He Y P, Zhang S H and Zhang J 2018 Tuning a layer to a three-dimensional cobalttris $\left(4^{\prime}\right.$-carboxybiphenyl)amine framework by introducing potassium ions Inorg. Chem. Commun. 90 65

32. Kurmoo M 2009 Magnetic metal-organic frameworks Chem. Soc. Rev. 381353 